\title{
A case of esophageal cancer with mesojejunal lymph node metastasis after total gastrectomy
}

\author{
Ayu Kato $\cdot$ Ken-ichi Mafune $\cdot$ Junko Kuroda $\cdot$ \\ Keisuke Kubota $\cdot$ Masashi Yoshida • \\ Keiichiro Ohta $\cdot$ Masaki Kitajima
}

Received: 14 May 2010/ Accepted: 1 November 2011/Published online: 20 November 2011

(C) The Author(s) 2011. This article is published with open access at Springerlink.com

\begin{abstract}
A 56-year-old man was diagnosed with esophageal cancer by upper gastrointestinal endoscopy for examination of dysphagia. The patient had undergone total gastrectomy and jejunal interposition 4 years previously for a gastric cancer at the pT1N0M0 stage according to the UICC-TNM classification. Enhanced CT findings revealed a 3-cm-diameter mass located near the superior mesenteric artery. We conducted subtotal esophagectomy associated with partial jejunectomy including mesojejunectomy. The mass was histologically diagnosed to be mesojejunal lymph node metastasis from esophageal cancer. Mesojejunal lymph node metastasis from esophageal cancer developing after total gastrectomy has been reported in only three cases including ours. The present lymph node metastases may have occurred via the newly developed lymphatic drainage route through the esophagojejunostomy, and this metastatic lymph node can be considered the regional lymph node. Therefore, resection of the interposed jejunal limb with mesojejunectomy may be rational in surgery on esophageal cancer developing after total gastrectomy.
\end{abstract}

Keywords Mesojejunal lymph node · Total gastrectomy

\section{Introduction}

The number of patients developing esophageal cancer after gastrectomy has increased. However, the route of lymphatic

A. Kato $(\bowtie) \cdot$ K. Mafune $\cdot$ J. Kuroda $\cdot$ K. Kubota $\cdot$

M. Yoshida $\cdot$ K. Ohta $\cdot$ M. Kitajima

Department of Surgery and Center for Digestive Diseases,

International University of Health and Welfare Mita Hospital,

1-4-3 Mita, Minato-ku, Tokyo 108-8329, Japan

e-mail: ayukato@iuhw.ac.jp drainage and the pattern of lymph node metastasis are unknown. In a remnant gastric cancer, patients with lymph node metastasis to the mesojejunum used for reconstruction are frequently reported. However, only a few cases of mesojejunal lymph node metastasis have been reported in an esophageal cancer after gastrectomy. The optimal extent of lymphadenectomy is controvertial. Herein, we report a case of esophageal cancer with mesojejunal lymph node metastasis following total gastrectomy with jejunal interposition.

\section{Case report}

A 56-year-old man consulted his primary physician for dysphasia. He had undergone total gastrectomy with lymph node dissection 4 years previously for gastric cancer, which had been pathologically diagnosed as pT1NOM0, stage IA ( $n=0 / 45$ ), according to the 6th edition of the UICC-TNM classification [1]. The reconstruction was jejunal interposition via the retro-colic route. He underwent upper gastrointestinal endoscopy and was referred to our hospital for an esophageal ulcerative lesion. Barium esophagography revealed a $5-\mathrm{cm}$, type 2 ulcerative lesion in the lower thoracic esophagus (Fig. 1). Upper gastrointestinal endoscopy showed a tumor occupied one third of the circumference of the esophagus. Pathological examination of the biopsy specimen revealed poorly differentiated squamous cell carcinoma. Abdominal computed tomography (CT) revealed a $3-\mathrm{cm}$ mass with a central low density area near the superior mesenteric artery (SMA) (Fig. 2).

Consequently, we diagnosed the tumor as a stage cT3N1M1b esophageal cancer according to the 6th edition of the UICC-TNM classification and performed subtotal esophagectomy with lymph node dissection and partial jejunectomy including mesojejunectomy. The right 
hemi-colon was used for esophageal reconstruction. The mass shown in the abdominal CT was a swollen lymph node located around the second branches of the second

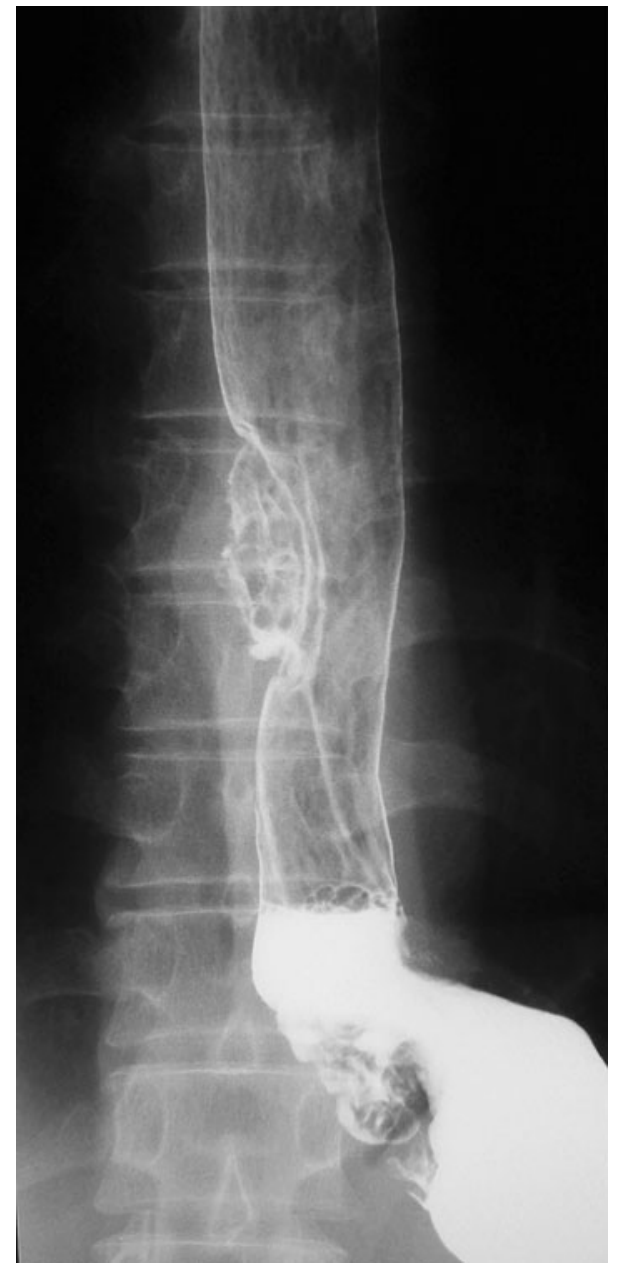

Fig. 1 Esophagography. Esophagography shows a type 2 tumor, $5 \mathrm{~cm}$ in length, in the lower thoracic esophagus

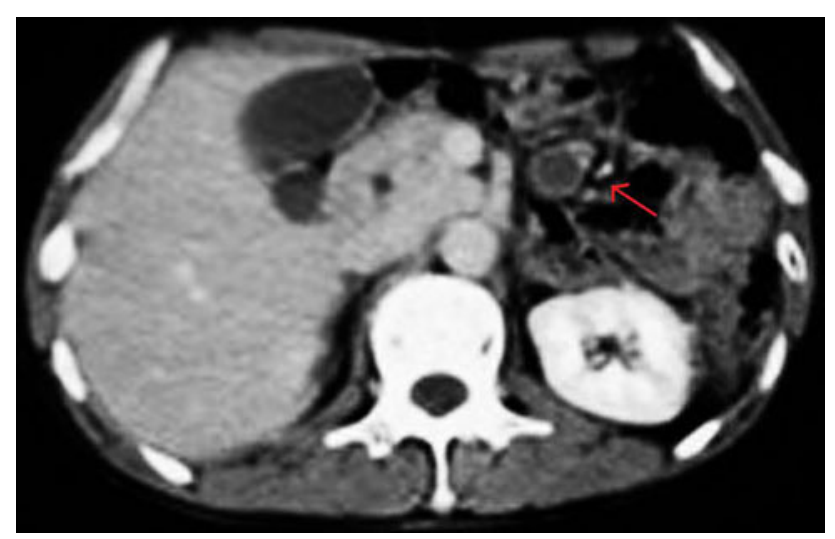

Fig. 2 Abdominal computed tomography (CT). Enhanced CT shows a tumor with central necrosis, $3 \mathrm{~cm}$ in diameter, near the superior mesenteric artery jejunal artery used for jejunal interposition. No swollen lymph node was observed along the marginal artery of the jejunum. Therefore, the $25-\mathrm{cm}$-long jejunum used for interposition was resected with the second branches of the second jejunal artery. The anal side of the right hemi-colon and the remaining jejunum interposition were anastomosed (Figs. 3, 4).

Histologically, the tumor was diagnosed as poorly differentiated squamous cell carcinoma, and metastasis was found in the subcarinal lymph node and the mesojejunal lymph node. The pathological stage was pT3N1 (2/37)M1b, stage IVB, according to the 6th edition of the UICC-TNM Classification of Malignant Tumors. Two courses of FP therapy (5 FU $960 \mathrm{mg} /$ body/day, days $1-5+$ CDDP $10 \mathrm{mg} /$ body/day, day 1) were administered as adjuvant chemotherapy, and then S-1 therapy (120 mg/body/day, 4 weeks administration and 2 weeks withdrawal) was performed for 2 years. No recurrence was observed for 5 years after the operation.

\section{Discussion}

The incidence of esophageal cancer after gastrectomy is $2.8-10 \%$ and has increased [2-4]. In an esophageal cancer after gastrectomy, abdominal lymphatic drainage routes are modified, but they are unknown.

In a remnant stomach cancer, metastasis to the mesojejunal lymph node is frequently observed. Especially in cases of Billroth II reconstruction with tumor invasion into the jejunum, the incidence of mesojejunal lymph node metastasis increased (9.1-55\%) [5-7]. Therefore, the Japanese Classification of Gastric Carcinoma prescribes describing whether invasion of a remnant stomach cancer into the

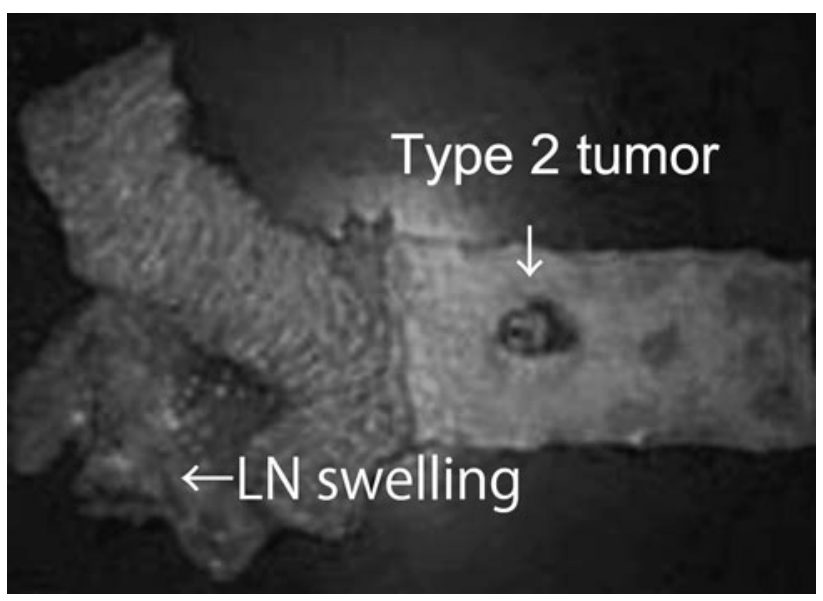

Fig. 3 Surgical specimen. A type 2 tumor is located in the lower thoracic esophagus. A swollen lymph node is observed along the second branch of the jejunal artery 


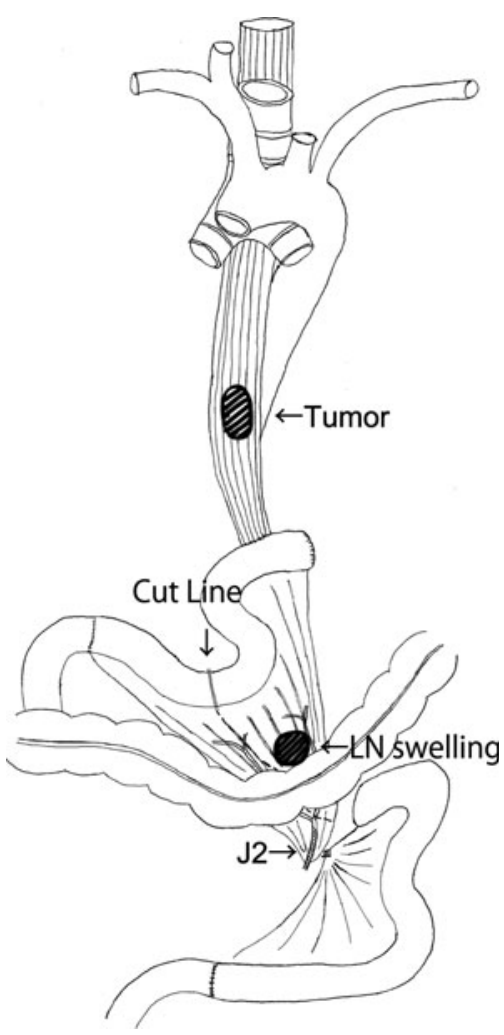

Fig. 4 Intraoperative findings. A swollen lymph node was located along the second branch of the jejunal artery $(J 2)$. We cut the second branch of the $\mathrm{J} 2$ and resected the $25-\mathrm{cm}$-long jejunum used for jejunal interposition

jejunum is observed or not [8]. In this case, mesojejunal lymph node metastasis occurred although invasion of the jejunum was not detected. Also, the metastatic lymph node was not at the marginal region of the jejunal interposition, but around the second branches of the jejunal artery.

Actually, in a remnant stomach cancer, lymphatic drainage into the jejunum and the colon was more frequently observed compared to a primary gastric cancer. In addition, there is a correlation between the lymphatic drainage and the incidence of lymph node metastasis [4]. Aiko et al. [3] reported six patients with abdominal lymph node metastasis around the remnant stomach or around the celiac artery among 20 cases of esophageal cancer after gastrectomy. In esophageal cancers after gastrectomy, only three cases with mesojejunal lymph node metastasis including the present case have been reported, and all the patients underwent total gastrectomy [9]. The reconstruction methods were the Rouxen-Y type in the other two cases.

In a remnant stomach cancer, lymphatic connection between the stomach and the jejunum is established through the anastomosis after the operation. Moreover, lymphatic connection with adjacent organs is established through the adhesion [10]. It is supposed that new lymphatic drainage routes between the esophagus and the jejunum arose through the anastomosis after total gastrectomy.

In an esophageal cancer, we can usually observe the lymphatic drainage routes between the lower esophagus and the abdominal aortic lesion. One is along the left gastric artery, and the other is along the left inferior phrenic artery. The lymph duct along the left inferior phrenic artery flows into the aortic lesion directly.

In the present case, we separated at the second branch of the jejunal artery and preserved the anal side of the jejunal interposition because the jejunum used for reconstruction in the previous operation was long enough. Should we remove all the jejunal interposition? How long should the jejunum resection be if the previous operation was a Roux-en-Y reconstruction? And should we perform lymph node dissection around the SMA? These many problems are still under consideration. In an advanced remnant stomach cancer, adequate resection of the jejunum and lymph nodes, especially lymph node dissection around the SMA, is important $[7,11]$. Some patients with a remnant stomach cancer showed a long survival after optimal lymph node dissection $[5,12,13]$. Also in an esophageal cancer after total gastrectomy, the mesojejunal lymph nodes may be regional lymph nodes, and therefore resection of the interposed jejunum used for reconstruction with mesojejunectomy will be required.

Open Access This article is distributed under the terms of the Creative Commons Attribution Noncommercial License which permits any noncommercial use, distribution, and reproduction in any medium, provided the original author(s) and source are credited.

\section{References}

1. Sobin L, Wittekind Ch, editors. TNM classification of malignant tumours. 6th ed. New York: Wiley-Liss; 2002.

2. Nishioka K. The promoter effect of GERD after gastrectomy on esophageal squamous carcino genesis. Ther Res. 2002;23:1035-42.

3. Aiko S, Yoshizumi Y, Sugiura Y, et al. Clinical characteristics of esophageal cancer after gastrectomy and the pertinence of chemoradiotherapy. J Jpn Surg Assoc. 2002;63:813-20.

4. Wada H, Doki Y, Nishioka K, et al. Clinical outcome of esophageal cancer patients with history of gastrectomy. J Surg Oncol. 2005;89:67-74.

5. Yonemura $\mathrm{Y}$, Tsugawa $\mathrm{K}$, Matsumoto $\mathrm{H}$, et al. Lymph node dissection for remnant stomach cancer. Surgery. 1993;47:75-82.

6. Kunisaki C, Shimada H, Nomura M, et al. Lymph node dissection in surgical treatment for remnant stomach cancer. Hepatogastroenterology. 2002;49:580-4.

7. Han SL, Hua YW, Wang CH, et al. Metastatic pattern of lymph node and surgery for gastric stump cancer. J Surg Oncol. 2003; 82:241-6.

8. Japanese Research Society for Gastric Cancer. Japanese classification of gastric carcinoma. 13th ed. Tokyo: Kanehara and Co. Ltd; 1999.

9. Ono T, Motoyama S, Saito R, et al. Mesojejunal lymph node metastasis in esophageal cancer following total gastrectomy. Jpn J Thorac Cardiovasc Surg. 2004;52:542-4. 
10. Hirose S. Experimental studies on the reparation of the lymph drainage of the stomach after removal of lymph nodes and lymphatics. Okayama J Med. 1965;77:1291-316.

11. Seto Y, Ohyama S, Nunobe S, et al. Open surgery for the remnant stomach cancer. Surg Ther. 2006;94:270-4.
12. Shoka J, Suzuki H, Kitamura Y, et al. Lymph node dissection in carcinoma of the remnant stomach. Jpn J Clin Sug. 1999;60:626-30.

13. Kosaka T, Ueshige N, Sugaya J, et al. A study on carcinoma of the gastric stump with special reference to lymph node dissection in the mesojejunum. Jpn J Gastroenterol Surg. 1999;32:972-7. 